\title{
Late within the Capsular Bag Intraocular Lens Dislocation (Ten - Year Experience)
}

\author{
Munir Amjad Baig ${ }^{1}$, Rabeeya Munir ${ }^{2}$ \\ ${ }^{1}$ Department of Ophthalmology, AJK Medical College, Muzaffar Abad, ${ }^{2}$ Islami Medical and Dental College
}

\begin{abstract}
Purpose: To find out the frequency and causes of within the bag intraocular lens dislocation.

Study Design: Descriptive, retrospective study.

Place and Duration of Study: Federal Government Services Hospital Islamabad, from 2008 to 2018.

Methods: Records of all the patients who underwent uneventful phacoemulsification were studied. Patients with Pseudoexfoliation and glaucoma were excluded. Out-of-the bag IOL dislocations and early dislocations that occurred within first three months after the cataract surgery were also not included. Percentage of patients with dislocated lens within the capsular bag after three months or more was calculated and the cause of dislocation was found. Results were presented in percentages.

Results: Three thousand patients underwent uneventful phacoemulsification. Two thousand nine hundred thirty two (2932) patients fulfilled the inclusion criteria while 68 patients did not return for follow up. Age ranged between 25 and 75 years. There were 1600 males and 1332 were females. One thousand seven hundred and sixty were right eyes and one thousand one hundred and seventy two were left eyes. Sixty one (2.08\%) developed late IOL dislocations, $35(57.3 \%)$ males and $26(42.7 \%)$ females. Patients of age group $50-75$ years had more IOL dislocations. Causes of dislocation included; advanced mature cataract $21.3 \%, 19.7 \%$ with postoperative trauma, uveitis $14.7 \%$, Myopia 9.8\%, Silicon plate design in 9.8\%, eccentric capsulorhexis $8.1 \%$, small capsulorhexis $6.5 \%$, retinitis pigmentosa $3.2 \%$ and in $6.5 \%$ no cause was found.
\end{abstract}

Conclusion: Advanced mature cataract and postoperative trauma were the commonest causes of IOL dislocation.

Key Words: Cataract surgery, Phacoemulsification, Trauma, Capsulorhexis. Intra ocular lens dislocation.

How to Cite this Article: Baig MA, Munir R. Late within the Capsular Bag Intraocular Lens Dislocation (Ten Year Experience). Pak J Ophthalmol. 2021, 37 (2): 179-182.

Doi: http://doi.org/10.36351/pjo.v37i2.1110

\section{INTRODUCTION}

With improved techniques, phacoemulsification with intra ocular lens (IOL) implantation has proved to be a successful and safe surgery for cataract extraction.

Correspondence: Munir Amjad Baig

Department of Ophthalmology, AJK Medical College, Muzaffar Abad

Email: drmuniramjad@gmail.com

Received: July 27, 2020

Accepted: January 26, 2021
Within the bag lens implantation is the gold standard for phacoemulsification. ${ }^{1,2}$ Apart from many known surgical complications like cystoid macular edema (CME), capsule shrinkage or rupture, posterior capsule opacification (PCO) and vitreous loss, dislocation of adequately placed IOL within the bag may occur any time within or more than three months of the safe surgery. ${ }^{3,4}$ IOL dislocations are termed as early and late depending on the time of occurrence..$^{5}$ Dislocations that occur within three months after surgery were categorized as early and after three months or many years after uneventful surgery were grouped as late. Late in the bag dislocation generally 
occurs as a result of zonular fiber weakness after uncomplicated surgery. ${ }^{6}$

The incidence of posterior chamber IOL dislocation is reported in the literature as $0.2 \%$ to3\%. ${ }^{7,8}$ The IOL dislocation risk after 10 years, 15 years, 20 years and after 25 years was $0.1 \%$, $0.2 \%, 0.7 \%$ and $1.7 \%$ respectively in a large retrospective, observational populationbased study. ${ }^{9}$

The rationale of this study is to find out the percentage of within the bag dislocation of IOL in a tertiary care hospital and to highlight the risk factors for dislocation.

\section{METHODS}

Records of patients with age $25-75$ years and operated for routine cataract surgery in Federal Government Services Hospital Islamabad were retrieved. Silicone and hydrophobic acrylic lenses manufactured with designs of both 1-piece and 3-piece were implanted. Patients with within the capsular bag IOL dislocation occurring three months after the safe surgery were included in this study. Two thousand nine hundred thirty two of total 3000 patients were followed up (97.7\%), 68 patients did not return for follow-up or reported dead. There were 1600 males and 1332 were females. The eye first operated was included in this study. The exclusion criteria was pseudoexfoliation and glaucoma patients, out-of-the bag IOL dislocation and early dislocations that occurred within first three months after the cataract surgery. Patient demographic data, surgery dates, lens material/design, capsular bag anomalies, YAG Laser and presence/absence of known risk factors were recorded. Detailed ocular examination was carried out by single surgeon which consisted of slit lamp examination, ophthalmoscopic examination, corrected visual acuity and intraocular pressure checkup. Data analyzed in frequencies/percentages.

\section{RESULTS}

In this study $61(2.08 \%)$ patients had developed late IOL dislocation, among them 35 (57.3\%) were males and $26(42.7 \%)$ were females. Average time between surgery and IOL dislocation was 5.8 years. During initial years, 2008 - 2012, the rate of IOL dislocation was high. Patients of $50-75$ years of age had more IOL dislocations. Advanced mature cataract and trauma were the most common associated conditions in this series.

\section{DISCUSSION}

Davison reported the first case of late spontaneous inthe-bag IOL dislocation in 1993 from capsular contraction syndrome. Between 1988 and 2001, 2663 IOLs were explanted and the cause for explanation in eight cases $(0.3 \%)$ was zonular dehiscence. ${ }^{10}$ The mechanisms of late IOL dislocation comprises of progressive zonular weakness and capsule contraction syndrome. Zonular weakness causes imbalance between centripetal and centrifugal forces over the capsular bag, resulting in dislocation. ${ }^{11}$

In the United States, the frequency of IOL dislocation ranges from $0.2-1.8 \%$ and is comparable to our study of $2.08 \% .{ }^{12}$ Clinically insignificant decentration occurs in $25 \%$ of cases and clinically significant decentration occurs in about $3 \%$ of the cases. $^{12}$ The mean interval of late in-the-bag dislocation has been estimated to be 7.5 years. In our study, it was 5.8 years. Krèpšte et al. ${ }^{13}$ reported that patients with lax zonules, advanced or mature cataracts and uveitis had shorter time interval. In this study, patients of 50 - 75 years age group developed IOL dislocation at an earlier stage. It was similar to another study, according to which zonular dehiscence and older age at cataract surgery were associated with a shorter interval between surgery and dislocation. ${ }^{14}$ Thirteen $(21.3 \%)$ patients having advanced mature cataract in our study developed late in-the-bag IOL dislocation similar to other study that mentioned its increased risk in advanced mature cataract. ${ }^{15}$

The most common condition associated with late IOL dislocation is pseudo-exfoliation syndrome (PEX) due to inadequate capsular and zonular support. 
Subjects with pseudo-exfoliation syndrome exhibit weak zonules by a process of elastinolysis. ${ }^{15}$ It also increases the anterior capsule contraction, which leads to zonular failure. In (PEX) IOL dislocation after cataract surgery had mean interval time of $5.5-8.5$ years. However, cases of pseudoexfoliation were excluded in our study.

Some surgeons had faced IOL dislocation 18 years after surgery. ${ }^{16}$ In this study $6(9.8 \%)$ myopic patients had IOL dislocation. Myopic eyes are large in all three dimensions (i.e. equatorial, Vertical and anteroposterior axis) resulting in zonular fibre elongation and zonular failure. ${ }^{17}$ In another study, high myopia as cause of dislocation of IOL was found in only $2.22 \% .^{18}$ In our study, most of the patients who had late IOL dislocation were males $35(57.3 \%)$ similar to some authors while according to other reports it was more common in women. ${ }^{19}$

The present study showed eccentric capsulorhexis in $4(6.5 \%)$ patients. A survey by Mamalis et $\mathrm{al}^{20}$ showed that an eccentric capsulorhexis might allow one of the lens edges to be more peripheral than the optic. In contrast, late decentration was due to subluxation associated with capsular fibrosis.

IOL dislocation/decentration is influenced by its material and design as well. Silicone-plate IOL design was prone to capsular contraction forces and may decenter, tilt or rotate. In our study $5(8.1 \%)$ patients developed IOL dislocation in which silicon plate design was used. However, some authors reported that any type of IOL was at risk for late in-the-bag IOL dislocation. ${ }^{21}$ History of postoperative trauma was present in $12(19.7 \%)$ patients in this study similar to Dabrowska et $\mathrm{al}^{22}$ who reported $11.1 \%$ of patients had history of trauma.

Østern et al $^{16}$ highlighted bilateral cases of IOL dislocation within the bag after many years of operation in $9.1 \%$ of the patients. Bilaterality has also been observed by other authors ${ }^{8}$ but was not consistent with our study which entails only first operated eyes.

Capsular tension ring CTRs was not used in any case in this study but reports reveal that it can prevent intraoperative zonular dehiscence and decrease postoperative capsule shrinkage. ${ }^{23}$ However, other authors have reported that period between original surgery and dislocation is shorter in cases where a capsular traction ring CTR was present within the capsular bag $(4.9 \pm 1.9$ years $){ }^{5}$

The continuous curvilinear capsulorrhexis (CCC) during phacoemulsification has decreased the rate of IOL dislocation. CCC supports the IOL optic for 360 degrees thus allowing good IOL fixation. ${ }^{24}$

Limitation of this study was retrospective design and single center study. Reports from other centers and other surgeon will give a true picture of frequency of within the bag dislocation of IOL.

\section{CONCLUSION}

The mechanism of late IOL dislocation comprises of progressive zonular weakness and capsule contraction syndrome. Zonular weakness results in balance between centrifugal and centripetal forces over the capsular bag, resulting in dislocation.

\section{Ethical Approval}

The study was approved by the Institutional review board/ Ethical review board. (ERB/AJKMC/Phy14/6).

\section{Conflict of Interest}

Authors declared no conflict of interest.

\section{REFERENCES}

1. Hirata A, Okinami S, Hayashi K. Occurrence of capsular delamination in the dislocated in-the-bag intraocular lens. Graefes Arch Clin Exp Ophthalmol. 2011; 249 (9): 1409-1415. Doi: 10.1007/s00417-0101605-5.

2. Pueringer SL, Hodge DO, Erie JC. Risk of late intraocular lens dislocation after cataract surgery, 1980-2009: A population-based study. Am J Ophthalmol. 2011; 152: 618-623.

3. Dabrowska-Kloda K, Kloda T, Boudiaf S, Jakobsson G, Stenevi U. Incidence and risk factors of late in-the-bag intraocular lens dislocation: evaluation of 140 eyes between 1992 and 2012. J Cataract Refract Surg. 2015; 41 (7): 1376-1382.

Doi: $10.1016 / j . j c r s .2014 .10 .040$.

4. Ganesh SK, Sen P, Sharma HR. Late dislocation of in-the-bag intraocular lenses in uveitic eyes: An analysis of management and complications. Indian $\mathbf{J}$ Ophthalmol. 2017; 65 (2): 148-154. Doi: 10.4103/ijo.IJO_938_16.

5. Steeples LR, Jones NP. Late in-the-bag intraocular lens dislocation in patients with uveitis. $\mathrm{Br} \mathrm{J}$ Ophthalmol. 2015; 99: 1206-1210. 
6. Kawano S, Takeuchi M, Tanaka S. Current status of late and recurrent intraocular lens dislocation: analysis of real-world data in Japan. JPN J Ophthalmol. 2019; 63: 65-72 https://doi.org/10.1007/s10384-018-0637-y

7. Gimbel HV, Condon GP, Kohnen T, Olson RJ, Halkiadakis I. Late in-the-bag intraocular lens dislocation: incidence, prevention, and management. J Cataract Refract Surg. 2005; 31: 2193-2204.

8. Fernández-Buenaga $\mathbf{R}$, Alio JL, Pérez-Ardoy AL, Larrosa-Quesada A, Pinilla-Cortés L, Barraquer R, et al. Late in-the-bag intraocular lens dislocation requiring explantation: Risk factors and outcomes. Eye (Lond). 2013; 27: 795-801.

9. Ford JR, Werner L, Owen L, Vasavada SA, Crandall A. Spontaneous bilateral anterior partial inthe-bag intraocular lens dislocation following routine annual eye examination. J Cataract Refract Surg. 2014; 40: $1561-1564$.

10. Davison JA. Capsule contraction syndrome. J Cataract Refract Surg. 1993; 19: 582-589.

11. Shingleton BJ, Yang Y, O'Donoghue MW. Management and outcomes of intraocular lens dislocation in patients with pseudoexfoliation. $\mathbf{J}$ Cataract Refract Surg. 2013; 39: 984-993.

12. Slade DS, Hater MA, Cionni RJ, Crandall AS. Ab externo scleral fixation of intraocular lens. J Cataract Refract Surg. 2012; 38: 1316-1321.

13. Krepste L, Kuzmiene L, Miliauskas A, Januleviciene I. Possible predisposing factors for late intraocular lens dislocation after routine cataract surgery. Medicina (Kaunas). 2013; 49 (5): 229-234.

14. Zetterberg M, Sundelin K, Stenevi U. Surgical repositioning of intraocular lenses after late dislocation: Complications, effect on intraocular pressure, and visual outcomes. J Cataract Refract Surg. 2013; 39 (12): 1879-1885.

15. Shingleton BJ, Yang Y, O'Donoghue MW. Management and outcomes of intraocular lens dislocation in patients with pseudoexfoliation. J Cataract Refract Surg. 2013; 39: 984-993.

16. Østern AE, Sandvik GF, Drolsum L. Late in-the-bag intraocular lens dislocation in eyes with pseudoexfoliation syndrome. Acta Ophthalmol. 2014; 92: 184-191.
17. Werner L, Zaugg B, Neuhann T, Burrow M, Tetz M. In the bag capsular tension ring and intraocular lens subluxation or dislocation. A series of 23 cases. Ophthalmology, 2012; 119: 266-271.

18. Laude A, Agrawal A. Spontaneous partial dislocation of an aphakic capsular bag in high myopia. J Cataract Refract Surg. 2011; 37 (2): 427-428.

19. Jakobsson G, Zetterberg M, Lundström M. Late dislocation of in-the-bag and out-of-the bag intraocular lenses: ocular and surgical characteristics and time to lens repositioning. J Cataract Refract Surg. 2010; 36: 1637-1644.

20. Mamalis N, Brubaker J, Davis D, Espandar L, Werner L. Complications of foldable intraocular lenses requiring explantation or secondary intervention--2007 survey update. J Cataract Refract Surg. 2008; 34 (9): 1584-1591.

21. Clark A, Morlet N, Ng JQ. Whole population trends in complications of cataract surgery over 22 years in Western Australia. Ophthalmology, 2011; 118: 10551061.

22. Dabrowska-Kloda K, Kloda T, Boudiaf S. Incidence and risk factors of late in-the-bag intraocular lens dislocation: evaluation of 140 eyes between 1992 and 2012. J Cataract Refract Surg. 2015; 41: 1376-1382.

23. Michaeli A, Soiberman U, Loewenstein A. Outcome of iris fixation of subluxated intraocular lenses. Graefes Arch Clin Exp Ophthalmol. 2012; 250 (9): 1327-1332.

24. Gonnermann J, Klamann MK, Maier AK, Rjasanow J, Joussen AM, Bertelmann E, et al. Visual outcome and complications after posterior irisclaw aphakic intraocular lens implantation. J Cataract Refract Surg. 2012; 38 (12): 2139-2143.

\section{Authors' Designation and Contribution}

Munir Amjad Baig; Associate Professor: Concepts, Design, Literature search, Dana Analysis, Manuscript editing, Manuscript review.

Rabeeya Munir; Demonstrator: Literature search, Data acquisition, Statistical analysis, Manuscript preparation, Manuscript editing. 